\title{
Response
}

\section{Independent Impact of Diabetes on the Severity of Coronavirus Disease 2019 in 5,307 Patients in South Korea: A Nationwide Cohort Study (Diabetes Metab J 2020;44:737-46)}

\author{
Sun Joon Moon ${ }^{1}$, Eun-Jung Rhee ${ }^{1}$, Won-Young Lee ${ }^{1}$, Kun-Ho Yoon ${ }^{2}$ \\ ${ }^{1}$ Department of Endocrinology and Metabolism, Kangbuk Samsung Hospital, Sungkyunkwan University School of Medicine, Seoul, \\ ${ }^{2}$ Department of Endocrinology and Metabolism, Seoul St. Mary's Hospital, College of Medicine, The Catholic University of Korea, Seoul, Korea
}

First of all, we would like to thank Kim et al. for their interest in our article, entitled "Independent Impact of Diabetes on the Severity of Coronavirus Disease 2019 in 5,307 Patients in South Korea: A Nationwide Cohort Study" [1]. We are pleased that Kim et al. recognized the value of our study with regard to future research.

In this Korean nationwide cohort study, we found that diabetes was associated with worse clinical outcomes in patients with coronavirus disease 2019 (COVID-19), including oxygen therapy, ventilator support, and mortality, independent of other comorbidities. Despite inconsistent results among studies from other countries, we suggested that our results originated from a relatively well-controlled and stable situation. To the best of our knowledge, our study was the first study using nationwide cohort from Korea that showed an independent impact of diabetes on the severity of COVID-19.

We generally agree with Kim et al's opinions on the limitations of our study and the need for further research. First, despite a high proportion of untreated diabetes patients (43.1\%), this is in line with the relatively high unawareness of diabetes (38.4\%) [2]. Since laboratory data are not available in the Kore- an Health Insurance Review and Assessment Service (HIRA) database, It is impossible to recognize these undiagnosed diabetes patients in this study. On one hand, among patients diagnosed with diabetes, 9.2\% were not receiving medical treatment [2]. Conversely, if diabetes is defined based on diagnostic codes to include these patients, some non-diabetic patients may be categorized as diabetic patients due to incorrect diagnostic codes, affecting accuracy. Therefore, this limitation appears inevitable when using HIRA data. Although some nondrug-treated patients with diabetes were classified as non-diabetes subjects, an independent impact of diabetes on the severity of COVID-19 was still seen in our study, so this could be considered a more conservative analysis of this association.

Second, Kim et al. suggested that it would be useful to consider the severity of diabetes in our study. Unfortunately, since laboratory data are not available in the HIRA database, diabetes severity could not be accurately assessed. We tried to perform subgroup analysis according to insulin use; however, logistic regression analyses were not suitable due to the small number of insulin users in this study. Obesity is an established risk factor for the severity of COVID-19 [3,4]. However, BMI
Corresponding authors: Won-Young Lee (D) https://orcid.org/0000-0002-1082-7592 Division of Endocrinology and Metabolism, Department of Internal Medicine, Kangbuk Samsung Hospital, Sungkyunkwan University School of Medicine, 29 Saemunan-ro, Jongno-gu, Seoul 03181, Korea

E-mail: drlwy@hanmail.net
This is an Open Access article distributed under the terms of the Creative Commons Attribution Non-Commercial License (https://creativecommons.org/licenses/by-nc/4.0/) which permits unrestricted non-commercial use, distribution, and reproduction in any medium, provided the original work is properly cited. 
data are not available in the HIRA data, and we believe that the diagnostic code for obesity is inaccurate because numerous obese patients do not receive treatment for obesity. Future research using other databases with laboratory and anthropometric data is needed.

Third, with regard to the loco-regional factor, we agreed with Kim et al's opinion that the outbreak in Daegu-Gyeongbuk and the shortage of healthcare resources in this area may have influenced the results. In our study, 59.4\% COVID-19 patients lived in this area. Since previous studies conducted in Daegu revealed an association between diabetes and mortality in COVID-19 patients in multivariable analyses, we would expect an independent impact of diabetes on the severity of COVID-19 to be found even after adjusting the location [5]. A further study addressing regional factors would be useful.

We would like to thank Kim et al. for their insightful commentary on our study. We hope that our study will act as a starting point for future research on the impact of diabetes on clinical outcomes of COVID-19.

\section{CONFLICTS OF INTEREST}

No potential conflict of interest relevant to this article was reported.

\section{REFERENCES}

1. Moon SJ, Rhee EJ, Jung JH, Han KD, Kim SR, Lee WY, Yoon $\mathrm{KH}$. Independent impact of diabetes on the severity of coronavirus disease 2019 in 5,307 patients in South Korea: a nationwide cohort study. Diabetes Metab J 2020;44:737-46.

2. Kim BY, Won JC, Lee JH, Kim HS, Park JH, Ha KH, Won KC, Kim DJ, Park KS. Diabetes fact sheets in Korea, 2018: an appraisal of current status. Diabetes Metab J 2019;43:487-94.

3. Jung CY, Park H, Kim DW, Lim H, Chang JH, Choi YJ, Kim SW, Chang TI. Association between body mass index and risk of COVID-19: a nationwide case-control study in South Korea. Clin Infect Dis 2020 Aug 25 [Epub]. https://doi.org/10.1093/ cid/ciaal257.

4. Cai Q, Chen F, Wang T, Luo F, Liu X, Wu Q, He Q, Wang Z, Liu Y, Liu L, Chen J, Xu L. Obesity and COVID-19 severity in a designated hospital in Shenzhen, China. Diabetes Care 2020; 43:1392-8.

5. Kim MK, Jeon JH, Kim SW, Moon JS, Cho NH, Han E, You JH, Lee JY, Hyun M, Park JS, Kwon YS, Choi YK, Kwon KT, Lee SY, Jeon EJ, Kim JW, Hong HL, Kwon HH, Jung CY, Lee YY, Ha E, Chung SM, Hur J, Ahn JH, Kim NY, Kim SW, Chang HH, Lee YH, Lee J, Park KG, Kim HA, Lee JH. The clinical characteristics and outcomes of patients with moderate-to-severe coronavirus disease 2019 infection and diabetes in Daegu, South Korea. Diabetes Metab J 2020;44:602-13. 